\title{
Diabetes: hora de rever as metas?
}

\author{
Diabetes: time to redefine goals?
}

Leandro Arthur Diehl'

\section{RESUMO}

Objetivo: O objetivo deste trabalho foi avaliar se a meta de A1c da Sociedade Brasileira de Diabetes (SBD) é compatível com as metas de glicemia propostas pelas mesmas diretrizes. Materiais e métodos: Um simulador computadorizado online (AIDA) foi usado para simular um paciente hipotético com os valores de glicemia iguais aos alvos propostos pela SBD. A glicemia média foi calculada a partir dos valores gerados pelo simulador e convertida para o valor correspondente de A1c usando-se a calculadora online da American Diabetes Association (ADA). Outros perfis, com diferentes níveis de glicemia, também foram simulados, para avaliar qual o valor de A1c correspondente a cada um dos perfis. Resultados: Os valores de glicemia de jejum $<100 \mathrm{mg} / \mathrm{dL}$, pré-prandiais $<110 \mathrm{mg} / \mathrm{dL}$ e pós-prandiais $<140 \mathrm{mg} / \mathrm{dL}$, recomendados pela SBD, geraram glicemia média de $123 \mathrm{mg} / \mathrm{dL}$, que correspondeu a A1c estimada de $5,9 \%$, muito abaixo da meta de $7 \%$ proposta pela SBD, mostrando incompatibilidade entre as metas de glicemia e A1c sugeridas nessa diretriz. Esse valor de A1c se associa a aumento do risco de morte em pacientes de alto risco. Conclusões: Além de recomendar valores diferenciados de A1c para diferentes tipos de paciente, a SBD também deveria discutir a adoção de metas diferenciadas de glicemia que fossem compatíveis com os níveis de A1c propostos, tornando mais claros os alvos de tratamento. Arq Bras Endocrinol Metab. 2013;57(7):545-9

\section{Descritores}

Diabetes melito/terapia; metas; hemoglobina A glicosilada; glicemia; simulação por computador

\section{ABSTRACT}

Objective: The aim of this study was to assess if the A1c goals from the Brazilian Diabetes Society (SBD) were compatible with their goals for blood glucose. Materials and methods: An online simulation (AIDA) was used to simulate a hypothetical patient with blood glucose values similar to the SBD's goals. Average glucose was calculated from generated blood glucose values, and then converted to the corresponding $\mathrm{A} 1 \mathrm{c}$ value, using the ADA online calculator. Other glycemic profiles, using different levels of blood glucose, were also simulated in order to assess which would be the A1c value associated with each profile. Results: Glycemic goals proposed by the SBD (fasting glucose $<100$ $\mathrm{mg} / \mathrm{dL}$, preprandial $<110 \mathrm{mg} / \mathrm{dL}$, and postprandial $<140 \mathrm{mg} / \mathrm{dL}$ ), were associated with an $\mathrm{A} 1 \mathrm{c}$ of $5.9 \%$, much lower than the goal of $7 \%$ recommended by the SBD. This demonstrates incompatibility among A1c and blood glucose goals proposed by the SBD. Such A1c levels are associated with increased mortality among high-risk patients. Conclusions: Besides recommending different A1c goals for different patients, the SBD should probably review its recommendations and adopt different blood glucose goals that are compatible with the proposed A1c goals, making therapeutic targets clearer. Arq Bras Endocrinol Metab. 2013;57(7):545-9

\section{Keywords}

Diabetes mellitus/therapy; goals; hemoglobin A glycosylated; blood glucose; computer simulation
1 Departamento de Clínica Médica, Centro de Ciências da Saúde (CCS), Universidade Estadual de Londrina (UEL), Londrina, PR, Brasil

Correspondência para: Leandro Arthur Diehl Departamento de Clínica Médica, Centro de Ciências da Saúde,

Universidade Estadual de Londrina Av. Robert Koch, 60 86038-440 - Londrina, PR, Brasil drgaucho@yahoo.com

Recebido em 24/Maio/2012 Aceito em 29/Maio/2013

\section{INTRODUÇÃO}

$\mathrm{E}$ m 2011, a Sociedade Brasileira de Diabetes (SBD) reviu suas diretrizes sobre o tratamento do diabetes melito (DM) em adultos, adotando novas metas de tratamento, a saber: glicemia de jejum $<100 \mathrm{mg} / \mathrm{dL}$, glicemias pré-prandiais < $110 \mathrm{mg} / \mathrm{dL}$ (sendo "toleráveis" valores até $130 \mathrm{mg} / \mathrm{dL}$ ), glicemias pós-prandiais $<140 \mathrm{mg} / \mathrm{dL}$ (sendo "toleráveis" valores até $160 \mathrm{mg} /$ dL) e Alc $<7 \%$ para adultos, com a recomendação de individualizar as metas de Alc conforme as características do paciente (1).

$\mathrm{Na}$ prática, temos observado frequentemente pacientes diabéticos em tratamento que apresentam glicemias fora da faixa proposta pela SBD (ou seja, maiores que $100 \mathrm{mg} / \mathrm{dL}$ em jejum, maiores que $110 \mathrm{mg} / \mathrm{dL}$ nos períodos pré-prandiais e/ou maiores que $140 \mathrm{mg} / \mathrm{dL}$ pós-prandiais), mas que mesmo assim mantêm valores de Alc dentro da meta de 7\%. Assim como nós, pro- 
vavelmente muitos médicos que tratam pacientes com DM devem encontrar-se, não raramente, com a seguinte dúvida: se o paciente está com a Alc dentro da meta, deve-se ajustar o tratamento até que todos os parâmetros glicêmicos estejam dentro das metas propostas pela SBD?

Além do mais, é difícil entender por que a meta de Alc deve ser individualizada de acordo com as características do paciente (tais como a duração do DM, a presença de comorbidades, a expectativa de vida do paciente etc.), sendo inclusive recomendados valores diferenciados de Alc a serem perseguidos em faixas etárias específicas (até $8 \%$ para idosos, por exemplo), enquanto a meta da SBD para glicemia de jejum é rígida $(<100$ $\mathrm{mg} / \mathrm{dL}$ ), "sem qualquer valor de tolerância extra", o que gera estranhamento, pois um paciente com metas mais altas de Alc deveria, consequentemente, ter metas mais altas de glicemia.

Diante dessas dúvidas, resolvemos simular o perfil de glicemia, ao longo de 24 horas, de um paciente diabético hipotético, de forma a contemplar todas as metas de glicemia propostas no último posicionamento oficial da SBD, para estimar qual seria a glicemia média e a Alc resultante, bem como simular diferentes perfis glicêmicos para descobrir qual o provável nível de Alc associado a cada um deles.

\section{MATERIAIS E MÉTODOS}

Para simular o perfil glicêmico de um paciente diabético hipotético, usamos o AIDA, um simulador online de acesso livre, com fins educacionais, amplamente estudado e testado, que está disponível na Web desde 1996. Ele se encontra disponível no endereço: http:// www.2aida.org/online/ (2). Usando o primeiro perfil (caso número 0001) dentre os vários existentes no AIDA, ajustamos sua medicação e suas refeições de forma que o paciente simulado apresentasse praticamente a totalidade dos valores de glicemia, ao longo das 24 horas, muito próximo (iguais ou, no máximo, $3 \mathrm{mg} / \mathrm{dL}$ acima ou abaixo) das metas definidas pela SBD - glicemia de jejum: $100 \mathrm{mg} / \mathrm{dL}$, glicemias pré-prandiais: $110 \mathrm{mg} / \mathrm{dL}$ e glicemias 2 horas após as refeições: $140 \mathrm{mg} / \mathrm{dL}$ (1).

A partir desse perfil, obtivemos os valores numéricos para as glicemias ao longo das 24 horas, gerados pelo próprio AIDA. Esses valores foram copiados para uma planilha Microsoft Office Excel 2007, para cálculo da média aritmética simples da glicemia durante esse intervalo de 24 horas. A média de glicemia obtida foi, então, alimentada numa calculadora que converte valores da glicemia média estimada (estimated Average Glucose - eAG) em valores de hemoglobina glicada Alc, e vice-versa, disponível livremente na página da American Diabetes Association (ADA), no endereço: http:// professional.diabetes.org/GlucoseCalculator.aspx (3).

$\mathrm{O}$ procedimento foi repetido para diversos perfis glicêmicos diferentes, sendo obtida a glicemia média esperada em cada um deles (no AIDA) e calculado o valor de Alc associado a essas glicemias médias (na calculadora da ADA).

\section{RESULTADOS E DISCUSSÃO}

O perfil montado no AIDA com glicemias muito próximas às estabelecidas nas metas da SBD gerou 97 valores de glicemia, ao longo das 24 horas (ou seja, uma medida a cada 15 minutos), que resultaram numa glicemia média de 122,87 $\pm 19,3 \mathrm{mg} / \mathrm{dL}$ (Figura 1). Essa glicemia média, informada à calculadora de $\mathrm{Alc} / \mathrm{eAG}$ da ADA, resultou num valor de Alc de 5,9\% (bem abaixo dos $7 \%$ que são propostos como meta pela SBD).

É interessante notar que o próprio simulador AIDA retornou, para esse mesmo perfil de glicemia, um valor de Alc de exatamente 7\%. Isso pode ser explicado pelo fato de que os valores de correlação entre glicemia média e Alc utilizados pelo simulador AIDA (construído em 1996) (2) e, provavelmente, também pela última diretriz da SBD (1), usam dados desatualizados, derivados da correlação entre glicemia e Alc observados no estudo Diabetes Control and Complications Trial (DCCT) $(4,5)$. Nesse estudo, uma Alc de $7 \%$ correspondia a uma glicemia média de $170 \mathrm{mg} / \mathrm{dL}$. No entanto, o DCCT, apesar de toda sua importância, não tinha como objetivo estudar a correlação entre glicemia média e Alc $(4,5)$.

Por outro lado, um estudo mais recente, o ADAG, que foi desenhado especificamente para avaliar a correlação entre eAG e Alc, mostrou que uma Alc de 7\% equivalia, na verdade, a uma glicemia média mais baixa $(154 \mathrm{mg} / \mathrm{dL}$, com intervalo de confiança de $95 \%$ : $123 \mathrm{a} 185 \mathrm{mg} / \mathrm{dL}$ ) (6). Esses novos valores foram imediatamente adotados pelas diretrizes mais recentes das sociedades internacionais de diabetes, como a ADA e a European Association for Study of Diabetes (EASD) $(7,8)$, como pode ser visto na tabela 1 . Por esse motivo, preferimos usar a calculadora online da ADA (que usa os valores do ADAG) para estimar o valor de Alc correspondente à glicemia média obtida na simulação no $\operatorname{AIDA}(3,6)$. 


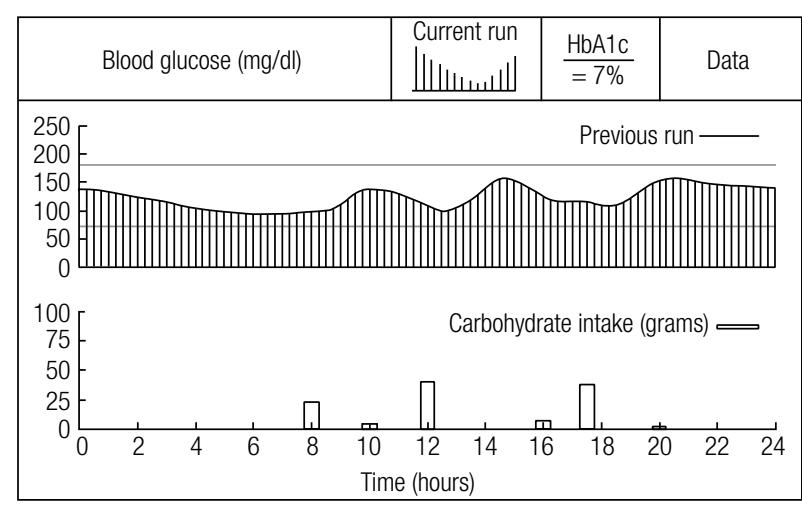

Figura 1. Gráfico do AIDA (2) mostrando as glicemias ao longo de um dia, em um paciente hipotético com valores glicêmicos dentro das metas propostas pelas diretrizes da SBD (1).

Tabela 1. Valores de glicemia média estimada (average Estimated Glucose - eAG) para cada valor de A1c, de acordo com os dados do DCCT e do ADAG

\begin{tabular}{lcc}
\hline A1c (\%) & eAG (DCCT, 1993) & eAG (ADAG, 2008) \\
\hline 5 & 100 & 97 \\
6 & 135 & 126 \\
7 & 170 & 154 \\
8 & 205 & 183 \\
9 & 240 & 212 \\
10 & 275 & 240 \\
11 & 310 & 269 \\
12 & 345 & 298 \\
\hline
\end{tabular}

Referências: DCCT (4,5); ADAG (6).

As diretrizes e guidelines das sociedades, construídas a partir da revisão crítica e sistemática das melhores evidências científicas disponíveis, são os documentos que norteiam o manejo atual de diversas patologias, inclusive o diabetes. Dessa forma, dada sua importância, parece-nos evidente a importância de essas diretrizes adotarem e recomendarem metas de controle glicêmico que sejam, a um só tempo: a) claras o suficiente para ser entendidas e aplicadas por não especialistas; b) internamente coerentes, de maneira que os valores de glicemia e Alc recomendados sejam compatíveis entre si e reflitam o mesmo grau de controle glicêmico; c) estritas o suficiente para reduzir o risco de complicações crônicas do DM a um nível satisfatório; e também: d) razoáveis, de maneira a não aumentar o risco de efeitos adversos decorrentes do próprio tratamento, especialmente hipoglicemia.

Quanto ao primeiro critério, observamos que as atuais diretrizes da SBD não são muito claras, pois, apesar de recomendarem a adoção de metas individualizadas de $\mathrm{Alc}$, não estabelecem metas diferenciadas para os valo- res da glicemia, em diferentes momentos do dia, como seria recomendável para atingir esses diferentes níveis de Alc. Além disso, as diretrizes não esclarecem, por exemplo, se há necessidade de intensificar o tratamento naqueles pacientes que mostrarem valores de glicemia fora das metas propostas, apesar de apresentar Alc dentro do alvo. Em relação ao segundo critério, observamos que as diretrizes também não são coerentes entre si, pois nossa simulação demonstrou que os valores de glicemia recomendados não são compatíveis com o valor de Alc preconizado.

Finalmente, ao avaliar o quarto e último critério, suscita preocupação o fato de que os valores de glicemia recomendados pelas diretrizes da SBD acabem correspondendo, na nossa simulação, a um valor de Alc inferior a 6\%. O estudo ACCORD foi um marco ao demonstrar que pacientes de alto risco cardiovascular, com perfil similar a boa parte dos pacientes DM vistos na prática clínica, quando tratados intensivamente para atingir uma meta de $\mathrm{Alc}<6 \%$ (próxima à obtida no nosso paciente simulado, $5,9 \%$ ), apresentavam aumento do risco de morte (9). Depois desse estudo, as sociedades internacionais passaram a recomendar metas individualizadas de Alc para pacientes com alto risco cardiovascular, alto risco de hipoglicemias, comorbidades graves ou curta expectativa de vida, de forma a não aumentar o risco de mortalidade associado ao controle glicêmico muito rigoroso $(7,8)$.

Portanto, à luz dessas considerações, acreditamos que seja chegada a hora de a SBD, cujas diretrizes norteiam o tratamento do DM em todo o território nacional, rever suas metas de controle glicêmico para adultos com DM, pois os valores de glicemia sugeridos na sua diretriz mais recente correspondem a um nível de Alc muito mais baixo $(5,9 \%)$ que a meta de Alc sugerida na mesma diretriz $(7 \%)$, podendo, quando empregados, aumentar o risco de hipoglicemias, principalmente em pacientes de alto risco.

Uma sugestão é a adoção das metas propostas pela ADA: glicemia de jejum (e pré-prandial) entre 70 e 130 $\mathrm{mg} / \mathrm{dL}$ e glicemias pós-prandiais < $180 \mathrm{mg} / \mathrm{dL}$, para pacientes "médios" (8). Esses valores são mais compatíveis com uma Alc próxima a 7\%, nível suficiente para reduzir significativamente o risco de complicações micro e macrovasculares em portadores de DM, com menor risco de hipoglicemias $(4,5,10)$.

Além disso, seria muito interessante se, além de recomendar diferentes metas de Alc, a SBD também recomendasse diferentes metas de glicemia (jejum, 
pré-prandial, pós-prandial), para diferentes grupos de pacientes, de forma que as metas de glicemia fossem coerentes com as metas de Alc. Para ajudar na seleção dessas diferentes metas de glicemia, mostramos na tabela 2 os resultados das simulações realizadas no AIDA com diferentes perfis glicêmicos (glicemia média e Alc associada a cada um dos perfis).

A tabela 3 ilustra, finalmente, nossa sugestão de metas de glicemia e Alc para diferentes perfis de pacientes, seguindo as sugestões de individualização de metas proposta pela ADA e EASD (11), e adotando valores de glicemia e Alc mais compatíveis entre si, com base nas simulações realizadas no AIDA (2).

Tabela 2. Valores de glicemia média e A1c para cada perfil glicêmico simulado no AIDA

\begin{tabular}{|c|c|c|c|}
\hline Perfil glicêmico (mg/dL) & Observação & $\begin{array}{c}\text { Glicemia } \\
\text { média } \\
\text { (mg/dL) }\end{array}$ & A1c (\%) \\
\hline $\begin{array}{l}\text { Glicemia jejum } 100 \\
\text { Glicemias pré-prandiais } 110 \\
\text { Glicemias pós-prandiais } 140\end{array}$ & $\begin{array}{c}\text { Metas da SBD, } \\
2011\end{array}$ & $123 \pm 19$ & 5,9 \\
\hline $\begin{array}{l}\text { Glicemia jejum } 100 \\
\text { Glicemias pré-prandiais } 130 \\
\text { Glicemias pós-prandiais } 160\end{array}$ & $\begin{array}{l}\text { Valores } \\
\text { "toleráveis" da } \\
\text { SBD, } 2011\end{array}$ & $129 \pm 20$ & 6,1 \\
\hline $\begin{array}{l}\text { Glicemia jejum } 110 \\
\text { Glicemias pré-prandiais } 110 \\
\text { Glicemias pós-prandiais } 160\end{array}$ & & $130 \pm 18$ & 6,2 \\
\hline $\begin{array}{l}\text { Glicemia jejum } 130 \\
\text { Glicemias pré-prandiais } 130 \\
\text { Glicemias pós-prandiais } 180\end{array}$ & $\begin{array}{c}\text { Metas da ADA, } \\
2012\end{array}$ & $142 \pm 22$ & 6,6 \\
\hline $\begin{array}{l}\text { Glicemia jejum } 140 \\
\text { Glicemias pré-prandiais } 140 \\
\text { Glicemias pós-prandiais } 180\end{array}$ & & $146 \pm 20$ & 6,7 \\
\hline $\begin{array}{l}\text { Glicemia jejum } 110 \\
\text { Glicemias pré-prandiais } 160 \\
\text { Glicemias pós-prandiais } 200\end{array}$ & & $162 \pm 32$ & 7,2 \\
\hline $\begin{array}{l}\text { Glicemia jejum } 140 \\
\text { Glicemias pré-prandiais } 140 \\
\text { Glicemias pós-prandiais } 200\end{array}$ & & $163 \pm 24$ & 7,3 \\
\hline $\begin{array}{l}\text { Glicemia jejum } 160 \\
\text { Glicemias pré-prandiais } 160 \\
\text { Glicemias pós-prandiais } 200\end{array}$ & & $170 \pm 17$ & 7,5 \\
\hline $\begin{array}{l}\text { Glicemia jejum } 140 \\
\text { Glicemias pré-prandiais } 180 \\
\text { Glicemias pós-prandiais } 220\end{array}$ & & $178 \pm 25$ & 7,8 \\
\hline $\begin{array}{l}\text { Glicemia jejum } 180 \\
\text { Glicemias pré-prandiais } 180 \\
\text { Glicemias pós-prandiais } 220\end{array}$ & & $188 \pm 19$ & 8,2 \\
\hline $\begin{array}{l}\text { Glicemia jejum } 180 \\
\text { Glicemias pré-prandiais } 180 \\
\text { Glicemias pós-prandiais } 250\end{array}$ & & $205 \pm 30$ & 8,8 \\
\hline
\end{tabular}

Referências: AIDA (2); SBD (1); ADA (8).
Tabela 3. Sugestão de metas de glicemia e A1c para diferentes perfis de paciente

\begin{tabular}{|c|c|c|c|}
\hline $\begin{array}{l}\text { Perfil de } \\
\text { paciente }\end{array}$ & $\begin{array}{c}\text { Controle mais } \\
\text { rigoroso }\end{array}$ & $\begin{array}{l}\text { Controle } \\
\text { "médio" }\end{array}$ & $\begin{array}{c}\text { Controle menos } \\
\text { rigoroso }\end{array}$ \\
\hline $\begin{array}{l}\text { Características } \\
\text { do paciente }\end{array}$ & $\begin{array}{l}\text { Jovem, hígido, } \\
\text { com curta } \\
\text { duração de } \\
\text { diabetes, sem } \\
\text { comorbidades } \\
\text { significativas, } \\
\text { motivado, boas } \\
\text { condições } \\
\text { socioeconômicas }\end{array}$ & $\begin{array}{l}\text { Características } \\
\text { intermediárias }\end{array}$ & $\begin{array}{l}\text { Idoso, com longa } \\
\text { duração de } \\
\text { diabetes, com } \\
\text { comorbidades } \\
\text { graves, curta } \\
\text { expectativa de } \\
\text { vida, alto risco de } \\
\text { hipoglicemias, } \\
\text { pouco suporte } \\
\text { social }\end{array}$ \\
\hline Meta de A1c & $6 \%$ & $7 \%$ & $8 \%$ \\
\hline $\begin{array}{l}\text { Meta de } \\
\text { glicemia de } \\
\text { jejum }\end{array}$ & $100^{*}$ & $130^{\#}$ & 160 \\
\hline $\begin{array}{l}\text { Meta de } \\
\text { glicemias } \\
\text { pré-prandiais }\end{array}$ & $110^{*}$ & $130^{\#}$ & 160 \\
\hline $\begin{array}{l}\text { Meta de } \\
\text { glicemias } \\
\text { pós-prandiais }\end{array}$ & $140^{*}$ & $180^{\#}$ & 200 \\
\hline
\end{tabular}

* Valores propostos pelas Diretrizes da SBD (1); ; Valores propostos pela ADA (8).

Como limitações do nosso trabalho, é importante citar que os valores de glicemia gerados em uma simulação computadorizada, por melhor que seja a simulação, provavelmente não levam em conta toda a complexidade de fatores que determinam o valor das glicemias de um paciente diabético real, ao longo de um único dia ou de vários dias, mas podem servir como aproximação bastante razoável, dado o grande número de variáveis e o amplo trabalho de pesquisa envolvido na sua construção (12). Além disso, a simulação foi intencionalmente ajustada, no nosso estudo, de forma a que o paciente hipotético apresentasse a grande maioria dos valores de glicemia dentro das metas estabelecidas, com estimativas da glicemia a cada 15 minutos, enquanto em pacientes reais frequentemente se observa maior variabilidade das glicemias, bem como menor frequência de verificação dos níveis de glicose plasmática. Mesmo assim, acreditamos que os resultados obtidos na nossa simulação possam servir como estímulo à discussão de metas glicêmicas mais coerentes entre si e com diferentes níveis de Alc.

\section{CONCLUSÕES}

Esperamos que nosso trabalho possa motivar a discussão de novas recomendações, que tragam, de maneira mais clara e individualizada, não só os valores de Alc, 
mas também os valores de glicemia a serem perseguidos como metas, nos diversos perfis de paciente. Tais recomendações provavelmente tornariam mais fácil, para os médicos que tratam DM (que são, na sua maioria, não especialistas), a tarefa de definir as metas de controle glicêmico mais adequadas a cada paciente específico, contribuindo para a melhoria do controle dos pacientes diabéticos como um todo e reduzindo o risco de efeitos adversos indesejáveis associados ao tratamento.

Declaração: os autores declaram não haver conflitos de interesse científico neste estudo.

\section{REFERÊNCIAS}

1. Sociedade Brasileira de Diabetes (SBD). Algoritmo para o tratamento do diabetes tipo 2 - atualização 2011. Posicionamento oficial SBD número 3 - 2011. Disponível em: http://www. diabetes.org.br/attachments/posicionamento/posicionamentosbd-n-03-2011.pdf

2. Lehmann ED, Deutsch T, Broad D. AIDA: and educational simulator for insulin dosage and dietary adjustment in diabetes. 1996. Disponível em: http://www.2aida.org/online/

3. American Diabetes Association. DiabetesPro - Professional Resources Online. Estimated Average Glucose (eAG) online calculator. Disponível em: http://professional.diabetes.org/ GlucoseCalculator.aspx

4. The effect of intensive treatment of diabetes on the development and progression of long-term complications in insulin-dependent diabetes mellitus. The Diabetes Control and Complications Trial Research Group. N Engl J Med. 1993;329:977-86.

5. DCCT Research Group: the association between glycaemic exposure and longterm diabetic complications in the Diabetes Control and Complications Trial. Diabetes. 1995;44:968-83.

6. Nathan DM, Kuenen J, Borg R, Zheng H, Shoenfeld D, Heine RJ, for the A1c-Derived Average Glucose (ADAG) Study Group. Translating the $\mathrm{A} 1 \mathrm{c}$ assay into estimated average glucose values. Diabetes Care. 2008;31:1-6.

7. Nathan DM, Buse JB, Davidson MB, Ferrannini E, Holman RR, Sherwin $\mathrm{R}$, et al. Medical management of hyperglycemia in type 2 diabetes: a consensus algorithm for the initiation and adjustment of therapy: a consensus statement of the American Diabetes Association and the European Association for the Study of Diabetes. Diabetes Care. 2009;32:193-203.

8. American Diabetes Association (ADA). Standards of medical care in diabetes - 2012. Diabetes Care. 2012;35(Suppl.1):S11-63.

9. The Action to Control Cardiovascular Risk in Diabetes (ACCORD) Study Group. Effects of intensive glucose lowering in type 2 diabetes. N Engl J Med. 2008;358:2545-59.

10. UK Prospective Diabetes Study (UKPDS) Group. Intensive bloodglucose control with sulphonylureas or insulin compared with conventional treatment and risk of complications in patients with type 2 diabetes (UKPDS 33). Lancet. 1998;352:837-53.

11. Inzucchi SE, Bergenstal SM, Buse JB, Diamant M, Ferrannini E, Nauck $M$, et al. Management of hyperglycemia in type 2 diabetes: a patient-centered approach - Position statement of the American Diabetes Association (ADA) and the European Association for the Study of Diabetes (EASD). Diabetes Care. 2012;35(6):1364-79.

12. Lehmann ED, Deutsch T. Compartmental models for glycaemic prediction and decision-support in clinical diabetes care: promise and reality. Comput Methods Programs Biomed. 1998;56:193204. 